\title{
Caries prevention for older people in residential care homes
}

\author{
Nicola Innes and Dafydd Evans \\ Preventive and Children's Dentistry Section, Dundee Dental Hospital and School, Dundee, Scotland, UK
}

When attempting to prevent dental caries in older people who live in residential care homes, what is the effectiveness and relative cost benefit of high concentration fluoride toothpaste compared with fluoride varnish application?

\section{Clinical scenario}

A dentist who is involved in oral health promotion is aware that dental caries is becoming more prevalent in an increasingly elderly population. ${ }^{1}$ He also knows that institutionalised elderly are an under-served group in terms of preventive oral health care. ${ }^{2,3} \mathrm{He}$ would like to improve the oral health of elderly residents of long-term care homes in the area where he works, in which water fluoride concentration is $<0.15 \mathrm{ppm}$.

The two interventions of interest to him are daily use of toothpaste containing a higher fluoride concentration than in normal paste for adults, and professionally applied fluoride varnish. His knowledge and understanding of caries, fluoride and the environment in which he would like to effect a change, lead him to postulate that these interventions might result in reduction of caries and subsequent development of dental pain and abscesses for the residents. He is uncertain, however, of the extent of any possible benefit and also whether using toothpaste with $>1450 \mathrm{ppm}$ fluoride would have a greater caries-protective effect than a fluoride varnish with 22,600 ppm fluoride. The dentist also wants to be able to discuss the relative cost of these two options, should they show any clinical effectiveness, when he presents his proposed caries-preventive programme for adoption. He decides to appraise the evidence for these questions by undertaking a literature review.

\section{Clinical question}

The following two clinical questions and their PICO (person/ intervention/ comparison/ outcome) questions were developed:

In elderly people who live in care homes (person), does the use of a) a high concentration (>1450 ppm fluoride) toothpaste (intervention) compared with standard concentration (1000-1450 ppm fluoride) toothpaste

\section{Table 1. Search strategy*}

\begin{tabular}{l|l|l}
\hline Search & Search term & Result \\
\hline 1 & caries AND NOT children & 25,334 \\
\hline 2 & elderly OR gerondont* OR aged OR geriatric & $2,816,726$ \\
\hline 3 & fluoride & 95,087 \\
\hline 4 & 1 and 2 and 3 & 327 \\
\hline 5 & Limit to: medicine and dentistry & 287 \\
\hline 6 & Limit to articles and reviews & 268 \\
\hline 7 & Limit to English & 250 \\
\hline *Scopus (1960 to date) was searched; last updated August 2009 (www.scopus.com/scopus/).
\end{tabular}

(comparison) or b) the use of biannual application of 22,600 ppm fluoride varnish (intervention) compared with no application of fluoride varnish (comparison), result in improved dental/ oral health (outcome) as measured by a reduction in caries experience, pain/ sepsis, improved aesthetics, and oral function?

What is the relative cost of each of these interventions?

\section{Search strategy}

The search strategy was based around the following key words:

- Dental caries, root caries, caries prevention, intervention programs

- Adult (population), elderly (population), aged, aged 80 and older, frail elderly, geriatric (population), gerodontology

- Long-term care facility, institutionalised/ institutionalized, care homes

- Toothpaste, topical fluorides, dentifrice, fluoride, fluoride gel, fluoride rinse, fluoride varnish, varnish, oral hygiene, professional tooth cleaning

\section{Document types: clinical trials} and reviews

All retrieved articles' titles were checked for relevance, and abstracts read where appropriate. If thought to be relevant, the papers were gathered and read. Six papers were considered appropriate and were subsequently appraised.

Additional searches for grey sources of information were carried out using CrossSearch and the Networked Digital Library of Theses and Dissertations. This revealed a recent $\mathrm{PhD}$ thesis from the University of Hong Kong which proved to be relevant but did not seem to have yielded any published papers as yet. The data were considered appropriate for inclusion in the review, as the complete thesis could be accessed and, having been passed at doctorate level, would have been peer reviewed.

\section{Discussion}

The vast majority of investigations for fluoride interventions to reduce dental caries have been conducted in children. Systematic reviews of these have unequivocally proven the effectiveness of topical fluoride in preventing/ arresting caries. ${ }^{10-13}$ There are few studies in the adult or elderly population, however. One systematic review confirmed that fluoride is an effective caries preventive measure in adults of all ages ${ }^{14}$ and a recent review of the effectiveness of fluoride 
interventions for prevention of root caries in adults found that regular topical fluoride was beneficial in reducing root caries. ${ }^{15}$

The dose-response relationship between fluoride concentration in toothpastes and reduction in dental caries was first reported in 1973 when it was shown that $1000 \mathrm{ppm}$ fluoride was more effective at reducing caries experience ${ }^{16}$ than 250 and 500 ppm fluoride. Since then, there have been many studies which have shown a positive relationship between fluoride concentration in dentifrices and other preparations (including mouthwashes and gel applications) and reduction in caries experience. Findings from the studies vary, but there is a consistent overall picture that higher fluoride levels have greater anticaries effects than lower fluoride levels. Again, most studies have been carried out in children. The few available studies of high fluoride concentration dentifrices (in excess of 2000 ppm fluoride), continue to support a linear dose-response relationship. ${ }^{17}$ An elderly population in a care facility presents a different group for a number of reasons including: less efficient metabolism, frequent health problems, polypharmacy, old teeth, established caries lesions, root caries, and an often limited ability to carry out oral healthcare themselves. This means that care must be taken when extrapolating the data from studies on children.

Six studies were identified that were relevant to the clinical question being asked and although not all were confined to elderly persons in residential care, all were in adults. Three of the studies compared high strength fluoride toothpaste (5000 ppm fluoride) to standard strength toothpaste (1000 ppm to $1450 \mathrm{ppm}$ fluoride). Two compared fluoride varnish $(22,600 \mathrm{ppm}$ fluoride) with standard toothpaste and one compared both high strength toothpaste and fluoride varnish with standard toothpaste. There are two main high strength toothpastes available in the UK (Duraphat 5000 ppm fluoride and Duraphat 2800 ppm fluoride; Colgate-Palmolive UK Ltd). No studies were found that had been carried out in adults using 2800 ppm fluoride toothpaste.

Although a variety of outcome measures were reported, all four toothpaste studies ${ }^{4-7}$ consistently found an improved response for caries control with the high strength fluoride toothpaste compared with standard toothpaste. This supports the dose-related response that is reported in the literature. ${ }^{17}$ No studies investigated what might be considered more patient-relevant factors such as aesthetics and oral function.

Three well-controlled randomised control trials compared fluoride varnish at 22,600 ppm fluoride (Duraphat varnish) with normal adult strength toothpaste (1000 ppm to 1450 ppm). ${ }^{7-9}$ All three studies reported an improved response for caries control with the varnish compared with the normal toothpaste (this was even the case in the one study ${ }^{9}$ that also included oral hygiene instruction in the control arm).

The effect of a high strength self-administered fluoride toothpaste (5000 ppm fluoride) and monthly hygienist applications of 22,600 ppm fluoride varnish were only directly compared in one study. ${ }^{7}$ Both interventions were found to be more effective at controlling root caries than brushing with $1450 \mathrm{ppm}$ fluoride toothpaste alone, but that there was no difference in the outcomes between the interventions. The 22,600 ppm fluoride varnish and the 5000 ppm fluoride toothpastes were equally effective but an additional basic cost analysis of the two interventions was carried out, which showed that the fluoride varnish was over 30-fold more expensive to provide, because of the cost of the hygienist.

For residents in care homes, there are additional considerations regarding potential excessive ingestion of fluoride. For adults, there are no UK recommendations on safe fluoride ingestion levels, but in the National Electronic Library for Medicines (www.nelm.nhs.uk) safe levels are taken from the a US committee (US Standing Committee on the Scientific Evaluation of Dietary Reference Intakes of the Food and Nutrition Board) that recommends that the tolerable upper limit for fluoride is $10 \mathrm{mg}$ a day for an adult. Older people, however, are more likely to have low body mass and to be frail. For residents in the area, drinking 1.5 litres a day of 0.15 ppm-fluoride-containing water would give a dietary intake of $0.225 \mathrm{mg}$ fluoride per day. In addition, they may drink large quantities of tea, especially if living in the warm, dry atmosphere of a care home. Tea can contain around 3 ppm fluoride, so if brewed from $0.15 \mathrm{ppm}$ fluoride water, drinking a litre a day of tea as a part of their 1.5 litres a day of fluids would give a total daily intake of $3.23 \mathrm{mg}$ fluoride per day. One gram of Duraphat $5000 \mathrm{ppm}$ fluoride paste will contain $5 \mathrm{mg}$ fluoride, and it is conceivable that an older person might swallow more excess paste than younger people, because of poor oral function or confusion. In addition, high turnover of staff is a fact of life in many care homes, so supervision of twice daily brushing might be less than adequate. It is also not an unlikely scenario that, given the high staff turnover in some long-term care homes, and the fact that the Duraphat 5000 ppm fluoride paste looks like a standard toothpaste, it might be used as such, in error. This would mean a full toothbrush of paste could be ingested in a frail elderly person twice a day. It would seem prudent, therefore, to recommend 2800 ppm fluoride paste for this population.

From a safety point of view, the use of Duraphat $(22,600 \mathrm{ppm}$ fluoride) varnish according to the manufacturer's instructions should not pose a toxicity hazard, and could be used on a 3-monthly basis as there is no risk of fluorosis in this population. It is an expensive product, though, and as it requires professional application, would be costly to apply.

In summary, there is good evidence that 22,000 ppm fluoride varnish and $5000 \mathrm{ppm}$ fluoride toothpaste are likely to be effective at reducing caries in an elderly population that is resident in care homes, in an area of low water fluoridation. With concerns regarding potential fluoride toxicity because of low body mass, poor oral function, uncertainty over level of supervision to avoid ingestion, and the quantity of daily fluid intake consumed as tea, it would seem prudent to recommend the use of $2800 \mathrm{ppm}$ fluoride paste, rather than the $5000 \mathrm{ppm}$ fluoride paste. The professional application of 22,600 ppm fluoride varnish three times a year could give additional benefit.

\section{Clinical bottom line}

For elderly populations in residential care, in areas with low levels of fluoride in drinking water, who are assessed as being at increased risk of dental caries:

- Toothbrushing carried out twice daily with a 2800 ppm fluoride paste, following the manufacturer's instructions regarding quantity, is likely to give improved caries control over standard toothpaste while avoiding any risk of toxicity. 


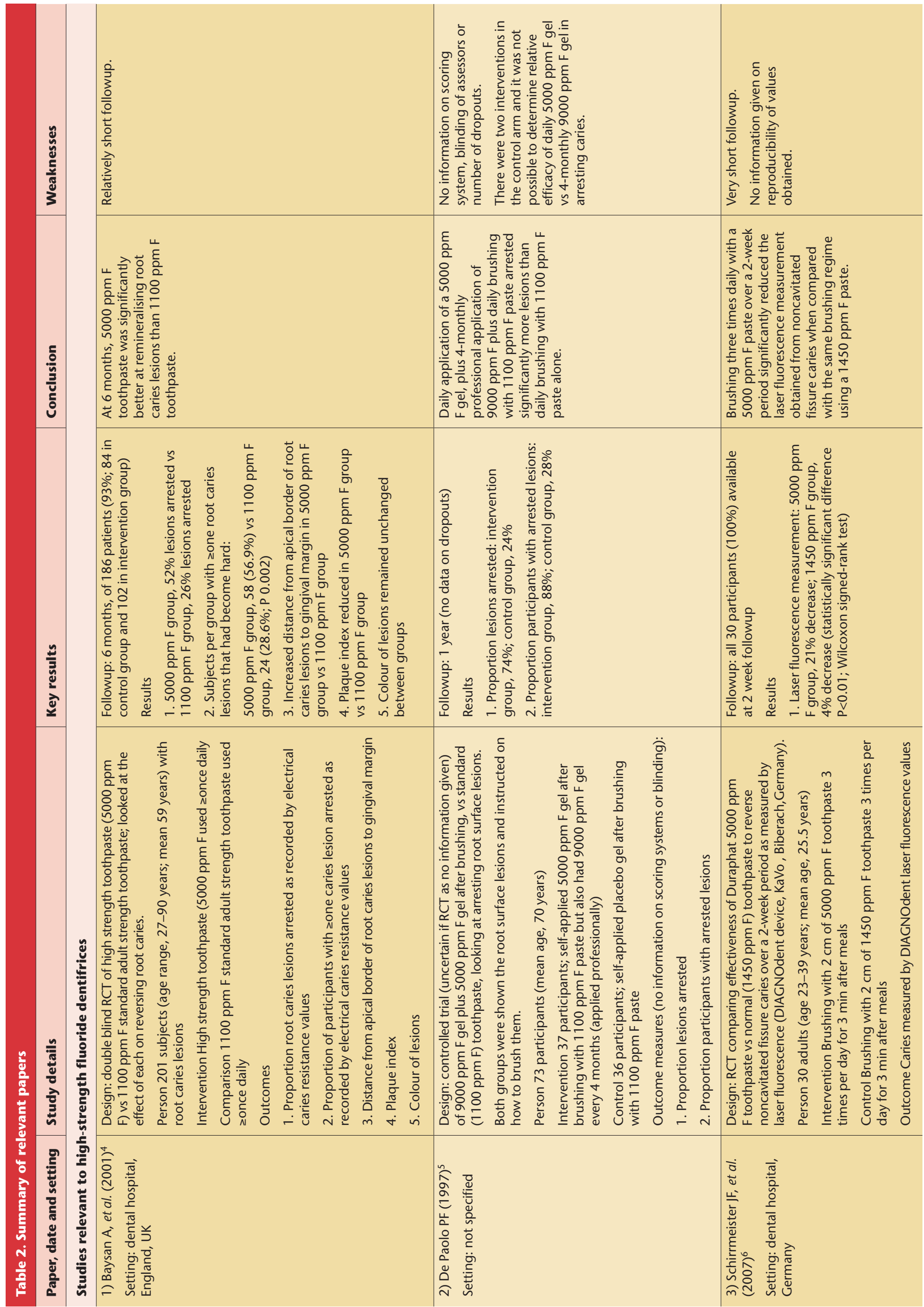




\section{DEBT}

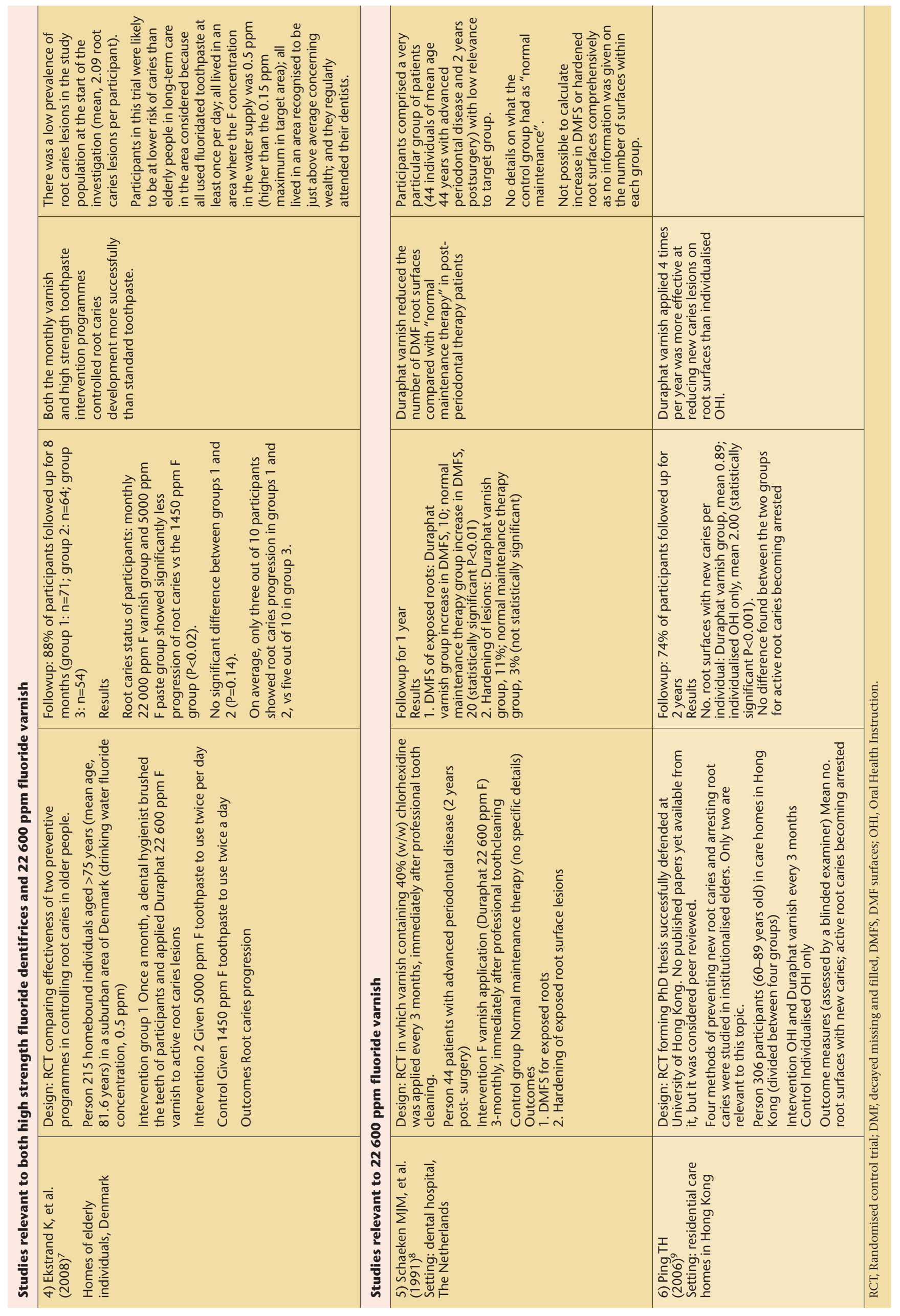


- On completion of brushing, the mouth should not be rinsed, but as much paste as possible should be expectorated.

- In addition, although costly, consideration could be given to applying 5\% sodium fluoride varnish to the same population three to four times per year.

1. Nunn J, Morris J, Pine C, Pitts NB, Bradnock G, Steele J. The condition of teeth in the UK in 1998 and implications for the future. Br Dent J 2000; 189: 639-644

2. Department of Health. Meeting the challenges of oral health for older people: a strategic review. Gerodontology 2005; 22 (suppl. 1): S3-S48.

3. Ellis AG. Geriatric dentistry in long-term-care facilities: current status and future implications. Special Care Dent 1999; 19: 139-142.

4. Baysan A, Lynch E, Ellwood R, Davies R, Petersson $L$, Borsboom P. Reversal of primary root caries using dentifrices containing 5,000 and 1,100 ppm fluoride. Caries Res 2001; 35: 41-46.
5. De Paola PF. The benefits of high-potency fluoride dentifrices. In Compendium of Continuing Education in Dentistry. 1997; 18 ( Spec No 2): S44-S50.

in Dentistry. 1997; 18 ( Spec No 2): S44-S50,
6. Schirrmeister JF, Gebrande JP, Altenburger MJ, Mönting JS, Hellwig E. Effect of dentifrice containing $5000 \mathrm{ppm}$ fluoride on non-cavitated fissure carious lesions in vivo after 2 weeks. Am I Dent 2007; 20: 212-216.

7. Ekstrand K, Martignon S, Holm-Pedersen P. Development and evaluation of two root caries controlling programmes for home-based frail people older than 75 years. Gerodontology 2008; 25: 67-75.

8. Schaeken MJM, Keltjens HMAM, Van Der Hoeven JS. Effects of fluoride and chlorhexidine on the microflor of dental root surfaces and progression of root-surface caries. J Dent Res 1991; 70: 150-153.

9. Tan H. Prevention and arrest of root surface caries in Chinese elders living in residential homes. PhD Thesis. Hong Kong: Hong Kong University; 2006.

10. Marinho VC. Evidence-based effectiveness of topical fluorides. Adv Dent Res 2008; 20: 3-7.

11. Marinho VC, Higgins JP, Sheiham A, Logan S. One topical fluoride (toothpastes, or mouthrinses, or gels, or varnishes) versus another for preventing dental caries in children and adolescents. Cochrane Database Syst Rev 2004, issue 1.
12. Marinho VC, Higgins IP, Sheiham A, Logan S. Combinations of topical fluoride (toothpastes, mouthrinses, gels, varnishes) versus single topical fluoride for preventing dental caries in children and adolescents. Cochrane Database Syst Rev 2004, issue 1.

13. Marinho VC, Higgins JP, Sheiham A, Logan S. Fluoride toothpastes for preventing dental caries in children and adolescents. Cochrane Database Syst Rev 2003, issue 1.

14. Griffin SO, Regnier E, Griffin PM, Huntley V. Effectiveness of fluoride in preventing caries in adults. J Dent Res 2007; 86: 410-415.

15. Heijnsbroek M, Paraskevas S, Van der Weijden GA. Fluoride interventions for root caries: a review. Oral Health Preventive Dent 2007; 5: 145-152.

16. Reed MW. Clinical evaluation of three concentrations of sodium fluoride in dentifrices. I Am Dent Assoc 1973; 87: 1401-1403.

17. Tavss EA, Mellberg JR, Joziak M, Gambogi R Fisher SW. Relationship between dentifrice fluoride concentration and clinical caries reduction. Am / Dent 2003; 16: 369-374.

Evidence-Based Dentistry (2009) 10, 83-87. doi:10.1038/sj.ebd.640672 